Therapie | Neuroanatomie

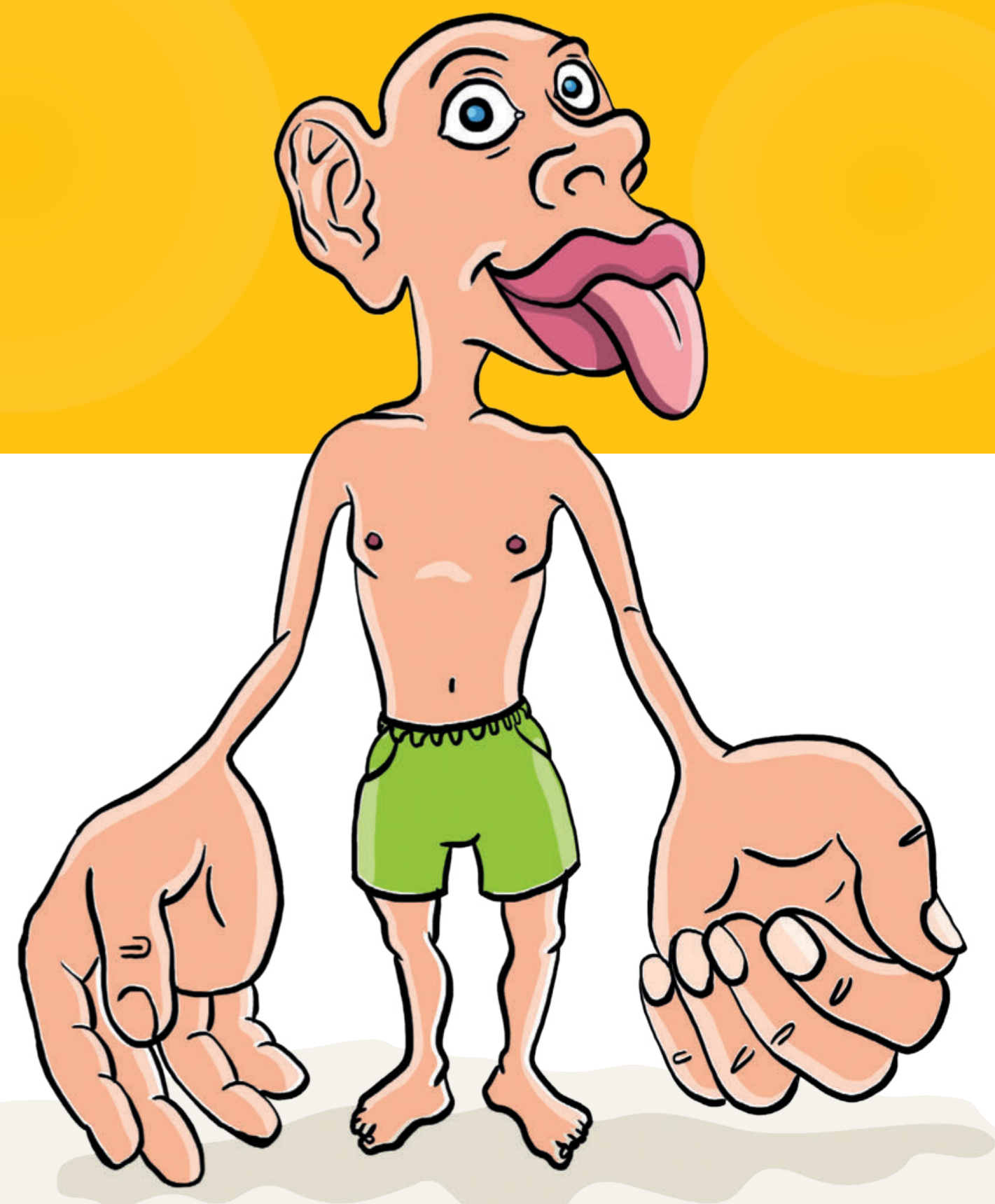

ABB. 1 Der Homunkulus ordnet die motorischen und somatosensorischen Areale der 


\title{
Das „Menschlein“ im Laufe der Zeit
}

\author{
Homunkulus 1950 erschien das erste Buch über ihn. Mit den unproportionierten Körperteilen, \\ den langen Fingern, den dicken Lippen und der großen Zunge hat der Homunkulus - lateinisch \\ für „Menschlein“ - einen bleibenden Eindruck hinterlassen. Bis heute repräsentiert er den Körper \\ des Menschen im Gehirn. Zeit, zu schauen, was sich über die Jahre bei ihm verändert hat.
}

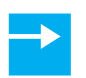

Der Homunkulus wurde erstmals 1937 von den beiden Neurochirurgen Wilder Penfield und Edwin Boldrey dargestellt. Er basierte auf den Ergebnissen elektrischer Hirnstimulationen auf den prä- und postzentralen Gyrus bei Patienten, die Penfield im Rahmen einer Epilepsieoperation untersucht hatte [1]. Der gewählte Ansatz war zeitaufwendig und mühsam. Einen ersten Homunkulus skizzierte Boldrey mithilfe von 170 Übersichtskarten aus Penfields Operationsnotizen, Fotos und Zeichnungen, die die Anzahl und Lage der Stimulationspunkte für die einzelnen Körperteile enthielten [1].

Mit diesem Meilenstein der Neurowissenschaft war erstmals die vollständige sensorische und motorische Landkarte des Körpers illustriert, und es ließen sich damit deren Funktionen innerhalb des menschlichen Gehirns lokalisieren. 1950 fasste Penfield sein Lebenswerk gemeinsam mit Theodore Rasmussen in einem Buch zusammen [2]. Dabei formierte die medizinische Zeichnerin Hortense Cantlie Penfields gesammelte Forschungsergebnisse zu dem Bild, das sich als Homunkulus in die Erinnerungen einprägen würde [3].

Der Homunkulus bildet die Reaktionen auf Hirnstimulationen für den Kopf, den Rumpf und die Extremitäten ab, die aus drei unterschiedlichen Maßen gewonnen wurden: die flächenmäßige Ausdehnung jedes Körperteils entlang der zentralen Furche auf dem prä- und postzentralen Gyrus, die Lokationen aller Stimulationen, die sich vor und hinter der Zentralfurche befinden, und die vertikale Ausdehnung jedes Körperbereichs entlang der Zentralfurche [4] (॰ ABB. 2 UND 3, S. 34 UND 35).

Obwohl Penfield und seine Kollegen die Ersten waren, die die vollständige kortikale Topografie des Körpers illustrierten, konnten sie auf Erkenntnissen früherer Untersuchungen aufbauen. Den entscheidenden Anreiz, nach der Lokalisation von Körperfunktionen im Hirn zu suchen, gab der französische Chirurg Pierre Paul Broca bereits 1861. Aufgrund von Läsionsstudien bei Patienten beschrieb er einen Bereich in der linken Hemisphäre des Menschen als spezialisiertes Sprachzentrum [5], heute als Broca-Areal bekannt.

Erste kontrollierte elektrische Stimulationen der Großhirnrinde führten die Mediziner Gustav Theodor Fritsch and Eduard Hitzig 1870 an der vorderen Hälfte an Hundehirnen durch und erzeugten Bewegungen von Muskelgruppen in der gegenüberliegenden Körperseite [6]. David Ferrier veröffentlichte 1876 ähnliche Ergebnisse mit wiederholten Hirnstimulationen an verschiedenen Säugetieren. Er erkannte, dass bestimmte Stimulationsorte vor der Zentralfurche Bewegungen erzeugen konnten [7]. Diese Erkenntnisse wurden zu Beginn des 20. Jahrhunderts im Gehirn von Affen bestätigt [8]. Charles Scott Sherrington und A. S. F. Grünbaum bestätigten 1902 erstmals die kortikale Organisation getrennter sensorischer und motorischer Regionen [9]. Schließlich war es der Neurochirurg Roberts Bartholow, der 1874 erstmals das menschliche Gehirn an einer wachen Patientin untersuchte, mit dem Ergebnis, dass die Probandin an den Folgen der Untersuchung starb [1, 10].

Diese frühen Experimente waren anspruchsvoll in der Ausführung, da die Reizung mit schwankender Stromintensität jäh einen Krampfanfall auslösen konnte. Schlussendlich aber überwogen die Vorteile, diese Methode bei Menschen einzusetzen, da nur sie Auskunft über ihre Empfindungen geben konnten. Den Anfang der elektrischen Hirnstimulation, deren Einsatz und Einfluss auf die Planung von Hirnoperationen sowie die damals vorherrschenden ethischen Grundsätze beschrieben Warren Boling und Kollegen 2002 in einer detaillierten Übersichtsarbeit [10].

Penfields Ergebnisse noch Jahre danach bestätigt $\rightarrow$ Auch nach 70 Jahren lässt das Thema die Wissenschaftler nicht los. Eine Untersuchung von 2020 bestätigt die Beständigkeit der kortikalen Somatotopik wie von Penfield beschrieben [11]. Der Neurochirurg FranckEmmanuel Roux hatte gemeinsam mit Kollegen den präzentralen 


\section{Therapie | Neuroanatomie}

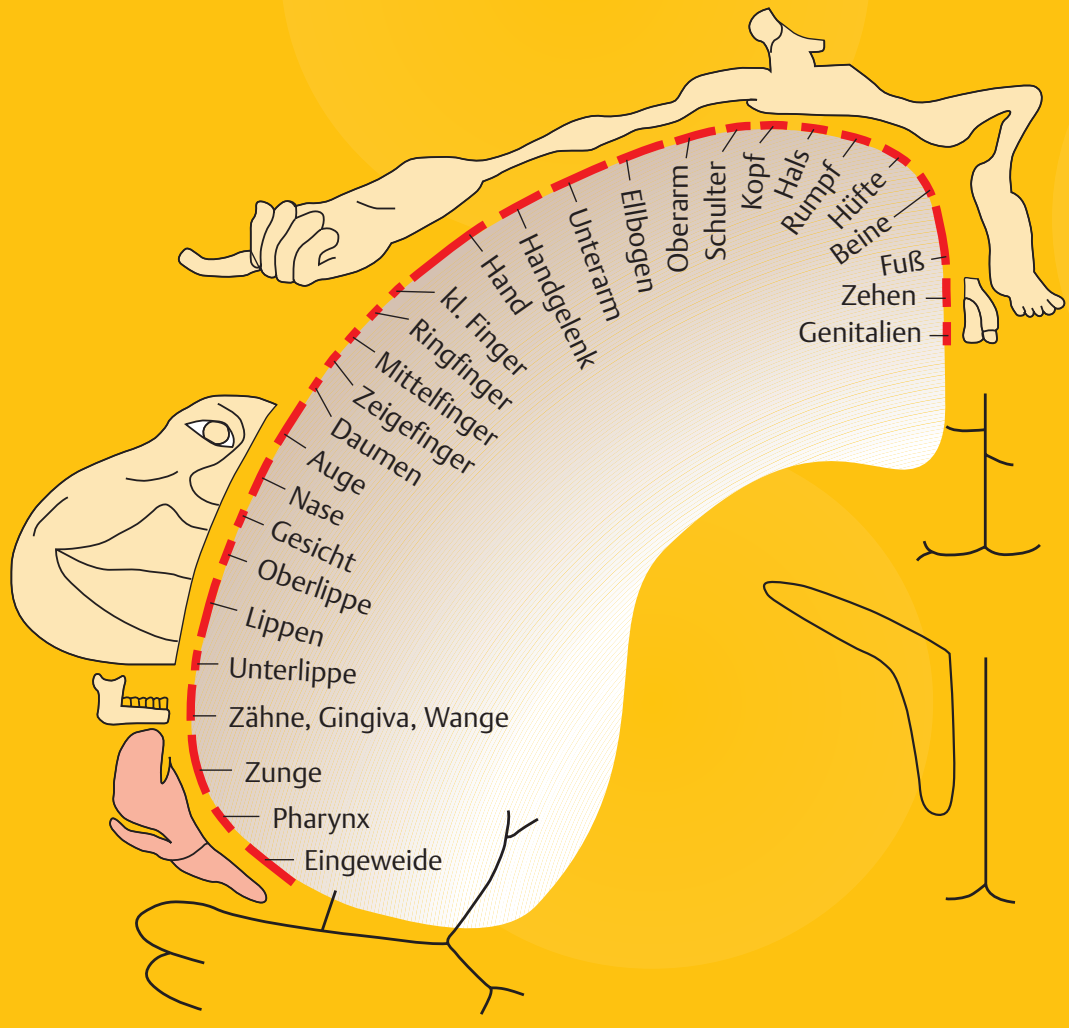

motorischen Kortex (M1) mittels elektrischer Stimulation während 100 Hirnoperationen untersucht und die Ergebnisse in ein Koordinatensystem transformiert, das üblicherweise für die Planung von Hirnoperationen verwendet wird. Die Replikation der Penfield'schen Ergebnisse ist darum beachtlich, weil Roux den Hirnstimulator mit moderner Neuronavigation platzieren und dabei auf stabile elektrische Frequenzen zählen konnte. Wobei auch in dieser Untersuchung die Autoren die äußerst heterogenen Intensitäten der Stimulationen, die für eine Aktivierung der Probanden benötigt wurden, herausstrichen. Zudem hatten die untersuchten Personen, im Unterschied zu Penfields Untersuchungen, keine organischen Läsionen im Bereich der Zentralfurche, womit die Forschenden eine mögliche Reorganisation in diesem Gebiet ausschließen konnten [12]. Schlussendlich konnten Roux und Kollegen die Repräsentation der Hand und der Kopf-Zungen-Region im Homunkulus verfeinern.

Die Körperoberfläche im sensorischen Homunkulus $\rightarrow$ Worauf aber basiert die somatotopische Abbildung des Körpers im sensorischen Homunkulus tatsächlich? Faktisch spiegelt der Homunkulus die topografische Anordnung der Körperteile wider, wobei das einem Körperteil gewidmete Areal des somatosensorischen Kortex (S1) im postzentralen Gyrus nicht proportional zu seiner tatsächlichen Größe abgebildet wird. Die Größe der Repräsentation basiert auf der Dichte peripherer Rezeptoren aus dem betreffenden Gebiet, die von Sinnesorganen und sensorischen Rezeptoren, wie die freien Nervenendigungen, Tast- und Lagesinn oder Druck, stammen [11]. Dabei stechen vor allem die Hand und der Mund-Gesicht-Bereich hervor, die wegen ihrer ausgeprägten diskriminativen Eigenschaften und kleinen rezeptiven Felder proportional viel größer dargestellt sind als ihre tatsächliche Hautoberfläche [13].

Die Größe der Repräsentation eines Körperteils im Homunkulus stimmt mit der Dichte der sensorischen Innervation überein, die mit der taktilen Schärfe bestimmt werden kann. Die taktile Schärfe wird als Präzision einer Berührung beschrieben und lässt sich mithilfe der Zwei-Punkte-Diskriminierung messen [14]. Diese wird über die Fähigkeit definiert, den kleinsten Abstand zwischen zwei taktilen Reizen, die an unterschiedlichen Punkten auf der Haut platziert werden, wahrzunehmen. Normative Werte der ZweiPunkte-Schwelle sind zum Beispiel in den Fingern bedeutend höher als am Rücken, was sich auch im Homunkulus deutlich zeigt $[13,16]$.

Genitalregion und Rumpf ungenau abgebildet $\rightarrow$ Man kann davon ausgehen, dass Körperteile mit hochqualifizierten Wahrnehmungsfunktionen nicht nur eine stärkere Innervation besitzen, sondern dass ihre Größe im Homunkulus auch die Spezialisierung der Funktion widerspiegelt [13]. Allerdings scheint diese Annahme für die Repräsentation der Genitalregion und des Rumpfes im sensorischen Homunkulus nicht zuzutreffen [17]. Bei genauer Betrachtung des sensorischen Homunkulus stellt man zudem eine Verletzung der topografischen Kontinuität bei der Lokalisation der männlichen Geschlechtsorgane fest. Warum sollten diese in der medialen Furche, unterhalb der Zehen repräsentiert sein? Natürlich war es aus anatomischen Gründen schwierig, diese Hirnregion zu untersuchen. Wahrscheinlicher aber haben historische Fragen des Anstands und der Scham, Empfindungen in solchen Regionen zu benennen, eine größere Rolle gespielt [18]. Dies könnte auch ein Grund dafür sein, dass Penfield die Somatotopik des Frauenkörpers in seinen Arbeiten kaum besprochen und in der Originalillustration nicht dargestellt hat [19]. Tatsächlich lokalisierten Untersuchungen mit funktionellem MRT die weiblichen und männlichen Geschlechtsorgane im unteren Bereich des Abdomens [18]. Dabei wurde auf deren sensorische Repräsentation verwiesen und jegliche erotische Wahrnehmung ausgeschlossen [17]. 


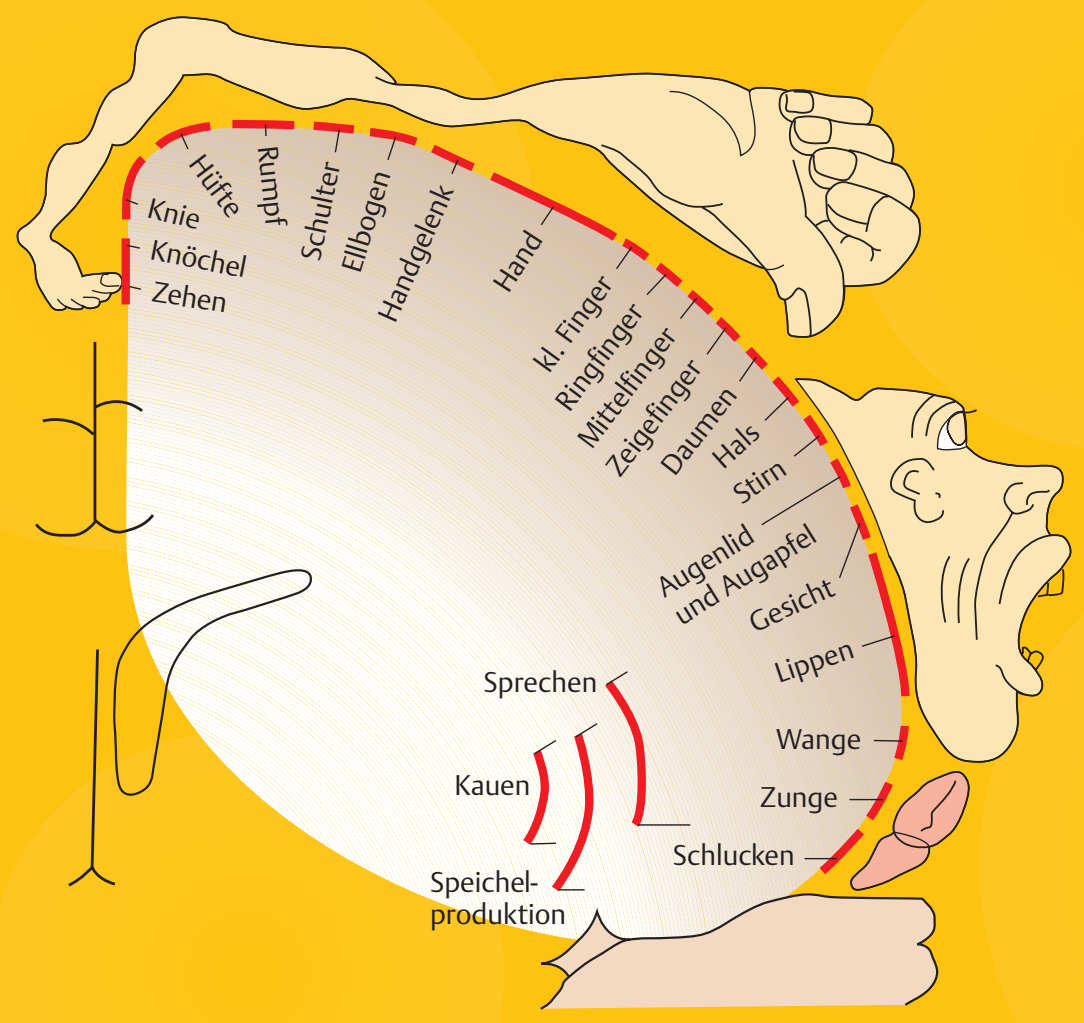

Eine weitere Ungereimtheit besteht in der unbedeutenden Darstellung des Rumpfs. Die visuelle Einprägung der Abbildung des Rumpfes hat zur Folge, dass man sie generell als klein ansieht. Diese Vorstellung mag stimmen, wird die geringe taktile Schärfe der Haut des Rumpfs betrachtet. Überlegt man sich aber, wie viele muskuloskelettale Strukturen für die vielfältigen Funktionen des Rumpfes verantwortlich sind, zum Beispiel Bewegung und/oder Stabilisation der Wirbelsäule, dann müsste die Repräsentation des Rumpfes in unseren Augen doch erheblich größer sein. Wir haben an der Universität Zürich daher mit funktionellem MRT passive posterioranteriore Bewegungen lumbaler Lendenwirbel untersucht und konnten dabei die topografische Lage des unteren Rumpfs an der Kante der Zentralfurche demonstrieren (॰ ABB. 4, S. 36) [20]. Als erstaunlich homogen erwiesen sich die Repräsentationen einzelner Wirbelkörper bei allen Versuchspersonen. Allerdings zeigten die Aktivierungsmuster im funktionel-

len MRT anders als im Homunkulus eine große Fläche der Repräsentation mit überlappender Innervation einzelner Wirbelkörper [20] (॰ ABB. 4, S. 36).

Der Homunkulus zeigt einzig die stabile somatotopische Lokalisation einzelner Körperteile $\rightarrow$ Multiple und überlappende Repräsentationen erkannte damals schon Penfield. Es verunsicherte ihn, was der Homunkulus tatsächlich repräsentieren würde [4]. Dies ist der Tatsache geschuldet, dass der postzentrale Gyrus (S1) in vier zytoarchitektonische Brodmann-Areale aufgeteilt ist (BA 1, 2, 3a und $3 b$ ) und jeder dieser Areale eine komplette somatotopische Projektion von bestimmten peripheren Haut-, Muskel- und Gelenk-

sensoren enthält [21]. Tatsächlich vermittelt die grafische Darstellung des Homunkulus einzig die äußerst stabile somatotopische Lokalisation einzelner Körperteile, während innerhalb der Körperregionen überlappende Repräsentationen gefunden wurden.

Zudem basiert der Homunkulus auf einer Momentaufnahme der sensorischen Empfindungen während einer Hirnstimulation und wird der generellen Komplexität der sensorischen Verarbeitung in keiner Weise gerecht. Die Rolle von S1 entspricht nämlich nicht der eines passiven Empfängers sensorischer Empfindungen, sondern der eines wichtigen Teils eines weit verbreiteten Netzwerks, das mit anderen Hirnarealen eng verbunden ist und in einem regen

95

Die Größe der Repräsentation basiert auf der Dichte peripherer Rezeptoren im betreffenden Gebiet. wechselseitigen Austausch steht [22]. Bildgebende Studien deuten darauf hin, dass S1 nicht nur sensorische Informationen verarbeitet, sondern auch an der taktilen Aufmerksamkeit beteiligt ist, vor allem wenn die zu verarbeitenden sensorischen Informationen von großer Bedeutung sind [23]. So stellt zum

Beispiel die taktile Schärfe keinen festen Wert dar, sondern kann durch die Beobachtung der Berührung verstärkt werden, nicht aber durch die bloße Darstellung des stimulierten Körperteils. Ähnliche Situationen sind im Alltag häufig anzutreffen und helfen, sensorische Funktionen zu vereinfachen, zum Beispiel beim Erkennen eines Objekts in der Hand [24]. Im Gegensatz dazu kann eine Immobilisation mit einem Gips dazu führen, dass die taktile Schärfe abnimmt [25].

Somatische Repräsentation der Körperteile scheint angeboren $\rightarrow$ Neben Informationen über ein externes Objekt vermittelt sensorischer Input gleichzeitig Informationen über den Körper selbst [26], 


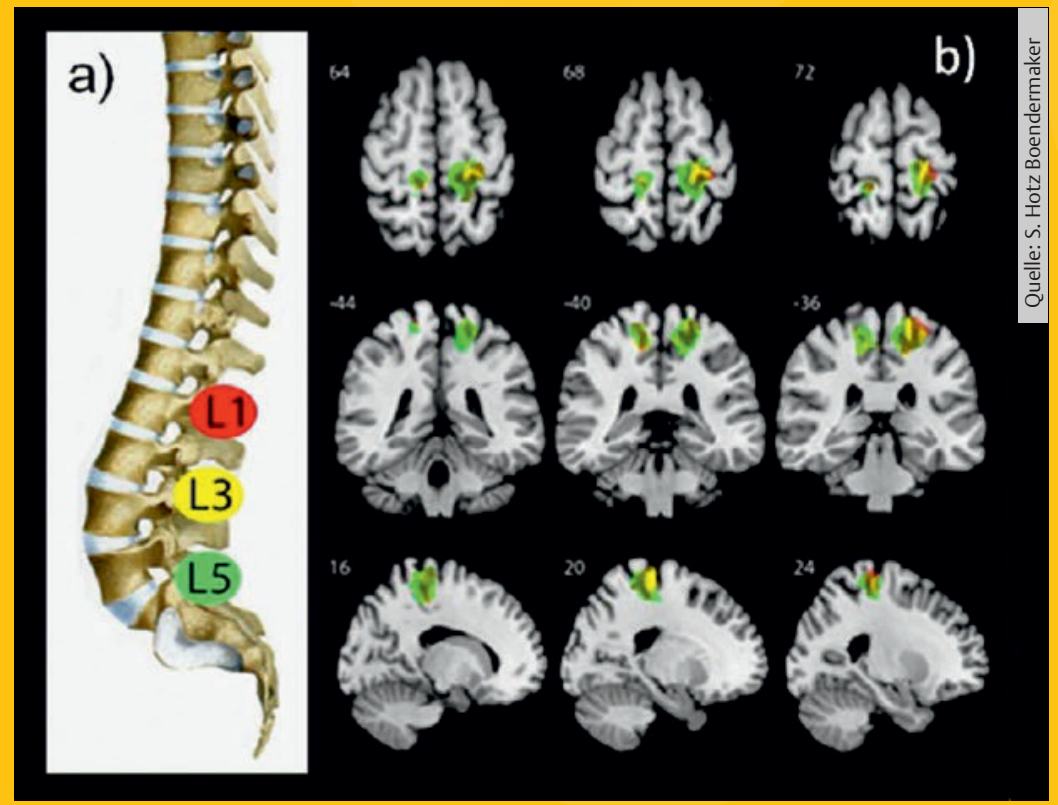

was zu einem frühen Konzept unseres eigenen Körpers und damit zum Selbst führt. Dabei wird eher das wahrgenommene Bild des Körpers codiert und weniger physische Informationen, die auf der Körperoberfläche wahrgenommen werden. Diese somatische Repräsentation der Körperteile scheint angeboren, wie eine Untersuchung einer Person zeigte, die ohne Arme und Beine geboren wurde, deren Extremitäten aber in sensorischen und motorischen kortikalen Arealen repräsentiert waren. Dabei scheinen sowohl genetische Faktoren als auch alltägliche Bewegungsbeobachtungen anderer Menschen zum bewussten Erleben von Phantomempfindungen in nicht vorhandenen Extremitäten zu führen [27].

Zu dem Beispiel passt, dass sich körperliche Erfahrungen schnell und einfach manipulieren lassen, zum Beispiel über visuellen Input. So sind nur wenige Schritte nötig, um künstliche Gliedmaßen in das Körperbild einzubinden, wie es Experimente mit der „Rubber Hand Illusion“ zeigen konnten [28, 29]. Dabei können komplexe Anforderungen die sensorische Repräsentation im Sinne einer funktionellen Plastizität in Sekundenschnelle durch zusätzlichen visuellen Input beeinflussen.

Auch wenn Wissenschaftler*innen jahrzehntelang an unveränderbare Verbindungen des Nervensystems glaubten, ist die neurale Plastizität heute allgemein anerkannt. Dabei kann es sich um eine ungünstige plastische Reorganisation handeln, wie im Fall von Phantomschmerzen. Ebenfalls dazu gehören aber auch vorteilhafte plastische Veränderungen als Folge von verhaltensrelevantem Training oder Lernen [30,31]. Plastische Veränderungen zeigen sich aber in jedem Fall in einer somatotopischen Reorganisation innerhalb von S1.

Motorischer Homunkulus hat Schlüsselfunktion bei der Koordination $\rightarrow$ Der motorische Homunkulus repräsentiert ein kortikales Areal im präzentralen Gyrus (primärer motorischer Kortex, M1), aus dem unwillkürliche Bewegungen durch Stimulation erzeugt werden können. Dabei sind die somatotopisch angeordneten Regionen des Kortex an der entsprechenden Kontrolle von Gesichts-, Arm- und Beinbewegungen beteiligt [12]. In frühen Tierexperimenten Anfang des 20. Jahrhunderts erzeugte eine Entfernung des postzentralen Gyrus (S1) keine Parese, während die Entfernung des präzentralen Gyrus (M1) eine Lähmung von Bewegungen zur Folge hatte, die zuvor durch elektrische Stimulation in diesem Areal erzeugt worden waren. Solche Experimente führten dazu, dass man davon ausging, dass der motorische Homunkulus eine Schlüssel-

\section{D)}

Die im motorischen und sensorischen Homunkulus definierten Hirnareale eines Körperteils tauschen sich aus. funktion bei der Codierung und Koordination der Aktivierungen peripherer Muskeln hat [32].

Aufgrund dieser Erkenntnisse wurde für die Handbewegung noch bis Mitte des 20. Jahrhunderts in M1 eine geordnete Punkt-zu-PunktRepräsentation der Bewegungen und Muskeln der Finger, des Handgelenks, des Ellenbogens und der Schulter angenommen, genau wie durch den Homunkulus vermittelt. Erst bildgebende Verfahren veränderten diese Sichtweise, indem demonstriert wurde, dass die Somatotopik der Armregion in M1 weder räumlich diskret noch sequenziell geordnet ist [33]. Vielmehr sind die Repräsentationen verschiedener Teile oder Muskeln der Hand jeweils weit innerhalb der Armrepräsentation verteilt, sodass sich die Repräsentationen zweier beliebiger kleinerer Anteile weitestgehend überlappen. Die Kenntnis dieser zugrunde liegenden Organisation ist wesentlich für das Verständnis des Beitrags von M1 zur Bewegungskontrolle [34]. 


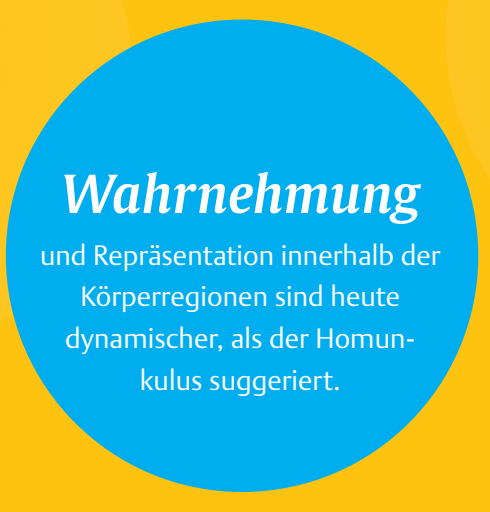

Wie der sensorische Homunkulus illustriert also auch der motorische Homunkulus nur die Oberfläche eines ausgedehnten Netzwerks. Dabei ist M1 über kurze Assoziationsbahnen wechselseitig mit kortikalen Arealen des Frontal- und Parietallappens verbunden, die für die Planung einer Bewegung verantwortlich sind. Für eine Greifbewegung muss zum Beispiel zuerst das angestrebte Verhalten definiert, die Position der Hand im Raum und im Verhältnis zum restlichen Körper bestimmt und die Stellung der Hand und der Finger erkannt werden, bevor in M1 schlussendlich die Handbewegung ausgelöst werden kann [35].

Die Bewegungsauslösung von M1 aus erfolgt über subkortikale Strukturen des Zwischenhirns, des Hirnstamms und der Motoneuronen des Rückenmarks, die über Projektionsbahnen Input von M1 erhalten [36]. Das vom motorischen Kortex ausgehende Projektionssystem ist jedoch nicht nur ein Effektororgan, sondern braucht für die Bewegungskontrolle ein dynamisches Rückkoppelungssystem. Auch wenn es auf den Darstellungen der Homunkuli nicht ersichtlich wird, befinden sich die jeweiligen, im motorischen und sensorischen Homunkulus definierten Hirnareale eines Körperteils in regem Austausch. Dies passiert durch wechselseitigen Input über eine Kette von U-förmigen Fasern unterhalb der Zentralfurche. Im menschlichen Gehirn sind diese U-förmigen Fasern in der Handregion besonders groß und nehmen in der ventralen Region der Handrepräsentation zunehmend an Volumen ab [4]. Diese reziproken Verbindungen zwischen S1 und M1 sind für die motorische Funktion essenziell.

Leider hat die individuelle Darstellung sensorischer und motorischer Homunkuli die Ansicht der Eigenständigkeit beider Systeme über Jahre zementiert. Bei einer Neubewertung des Homunkulus sollte man M1 daher als Rechnungszentrale innerhalb eines ausgedehnten Netzwerks kortikokortikaler und kortikosubkortikaler Verbindungen betrachten, das der Umsetzung von Bedürfnissen in Handlungen dient.
Heute wäre der Homunkulus flexibler $\rightarrow$ Der Penfield'sche Homunkulus hat als gut merkbare Gedankenstütze für die Repräsentation des Körpers im Hirn eine große Popularität erlangt und wird aus diesem Grund seinen Platz in den neurologischen Lehrbüchern behalten. Er illustriert die äußerst stabile motorische und sensorische Repräsentation einzelner Körperteile im prä- und postzentralen Gyrus, ein Faktum, das sich in unzähligen Untersuchungen bis in die heutige Zeit bestätigt hat. Allerdings ist die Wahrnehmung und Repräsentation innerhalb der einzelnen Körperregionen dynamischer und flexibler, als es die Darstellung des Homunkulus suggeriert. Zudem pflegen die motorischen und sensorischen Areale einen regen Informationsaustausch. Das Gehirn braucht Vielfalt und Flexibilität in seiner Organisation, um sich an schnell wechselnde Umweltsituationen anzupassen und zu lernen - dafür ist das Gehirn gut aufgestellt.

Sabina Hotz Boendermaker

$\rightarrow$ Literaturverzeichnis

www.thieme-connect.de/products/ergopraxis > "Ausgabe 1/22“

\section{Autorin}

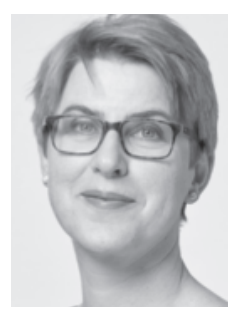

Dr. Sabina Hotz Boendermaker ist Physiotherapeutin und Psychologin. Sie arbeitet als wissenschaftliche Mitarbeiterin und Dozentin für Schmerzpsychologie an der Zürcher Hochschule für Angewandte Wissenschaften. Ihre Arbeits- und Forschungsschwerpunkte liegen unter anderem in den Bereichen Schmerzpsychologie, Aktivitätsmuster bei Rückenschmerzen sowie neurale Repräsentation von Schmerzen. 